\title{
Interpolation of Coefficients and Transformation of the Dependent Variable in Finite Element Methods for the Non-linear Heat Equation
}

\author{
Stig Larsson \\ Department of Mathematics, University of Michigan, 347 West Engineering Building, Ann Arbor, \\ MI 48109-1092, U.S.A. and Department of Mathematics, Chalmers University of Technology, \\ $S-41296$ Göteborg, Sweden \\ and \\ Vidar Thomée and Nai-Ying Zhang \\ Department of Mathematics, Chalmers University of Technology, S-41296 Göteborg, Sweden \\ Communicated by W. Wendland
}

Error estimates are shown for some spatially discrete Galerkin finite element methods for a non-linear heat equation. The approximation schemes studied are based on the introduction of the enthalpy as a new dependent variable, and also on the application of the Kirchhoff transformation and on interpolation of the non-linear coefficients into standard Lagrangian finite element spaces.

\section{Introduction}

In this paper we study semidiscrete finite element methods with interpolated coefficients for the non-linear heat equation

$$
\begin{array}{rlrl}
c(u) u_{t}-\nabla \cdot(a(u) \nabla u) & =f(u), & & \text { in } \Omega \times(0, T), \\
u=0, & & \text { on } \partial \Omega \times(0, T), \\
u(\cdot, 0)=v, & & \text { in } \Omega,
\end{array}
$$

where $\Omega$ is a bounded polygonal domain in $\mathbb{R}^{d}$ with $d \leqslant 3$. For the spatial discretization of (1.1) we shall consider standard piecewise polynomial Lagrangian finite element spaces. Thus, we denote by $S_{h}$ the space of continuous functions on $\Omega$ that reduce to polynomials of degree $\leqslant r-1$ on each simplex of a triangulation of $\Omega$. We seek approximate solutions to (1.1) in the subspace $S_{\text {oh }}$ consisting of those functions in $S_{h}$ that satisfy the boundary condition in (1.1). See Section 2 for the precise statements 
of our assumptions about the initial-boundary value problem (1.1) and the finite element spaces to be studied.

Consider first the standard semidiscrete Galerkin finite element method of finding $u_{h}:[0, T] \rightarrow S_{0 h}$ such that

$$
\begin{aligned}
\left(c\left(u_{h}\right) u_{h, 1}, \chi\right)+\left(a\left(u_{h}\right) \nabla u_{h}, \nabla \chi\right) & =\left(f\left(u_{h}\right), \chi\right) \text { for } \chi \in S_{0 h}, 0<t<T, \\
u_{h}(0) & =v_{h},
\end{aligned}
$$

where $(\cdot, \cdot)$ denotes the usual inner product in $L_{2}(\Omega)$ and $v_{h} \in S_{0 h}$ is an approximation of $v$. In terms of the standard Lagrangian nodal basis $\left\{\phi_{i}\right\}_{i=1}^{N_{h}}$ of $S_{0 h}$ this reads

$$
\begin{aligned}
\mathbf{C}(\mathbf{U}) \mathbf{U}^{\prime}+\mathbf{A}(\mathbf{U}) \mathbf{U} & =\mathbf{F}(\mathbf{U}), \text { for } 0<t<T, \\
\mathbf{U}(0) & =\mathbf{V},
\end{aligned}
$$

where $\mathrm{U}=\left(U_{i}\right)$ and $\mathrm{V}=\left(V_{i}\right)$ are the vectors of nodal values of $u_{h}$ and $v_{k}$, respectively, and $\mathbf{C}(\mathbf{U})$ is the non-linear mass matrix with entries $\mathbf{C}(\mathbf{U})_{i j}=\left(c\left(\Sigma_{k} U_{k} \phi_{k}\right) \phi_{i}, \phi_{j}\right)$. Similarly, the non-linear stiffness matrix $\mathbf{A}(\mathbf{U})$ is given by $\mathbf{A}(\mathbf{U})_{i j}=\left(a\left(\Sigma_{k} U_{k} \phi_{k}\right)\right.$ $\left.\nabla \phi_{i}, \nabla \phi_{j}\right)$ and the right-hand side $\mathbf{F}(\mathbf{U})$ by $\mathbf{F}(\mathbf{U})_{j}=\left(f\left(\Sigma_{k} U_{k} \phi_{k}\right), \phi_{j}\right)$.

From the point of view of actually computing the solution we note two difficulties: (i) the system is not written in normal form $Y^{\prime}=f(t, Y)$, and (ii) the above inner products must be computed by numerical quadrature. The first difficulty can be handled by a classical transformation of the dependent variable. With $H(u)=$ $\int_{0}{ }_{0} c(s) \mathrm{d} s$ - the enthalpy-and $G(u)=\int_{0}^{u} a(s) \mathrm{d} s$-the Kirchhoff transformation-the differential equation in the semidiscrete problem (1.2) can be written

$$
\left(H\left(u_{h}\right)_{i}, \chi\right)+\left(\nabla G\left(u_{h}\right), \nabla \chi\right)=\left(f\left(u_{h}\right), \chi\right) .
$$

For the numerical quadrature we shall replace the coefficients by their interpolants. Thus, let $I_{h}$ be the operator which associates with each continuous function $g$ its interpolant $I_{h} g \in S_{h}$ defined by $\left(I_{h} g\right)(P)=g(P)$ for each of the nodes $P$ that define the degrees of freedom of $S_{k}$. We are then led to consider the following interpolated coefficient finite element method: find $u_{h}:[0, T] \rightarrow S_{0 h}$ such that

$$
\begin{aligned}
\left(I_{h} H\left(u_{h}\right)_{l}, \chi\right)+\left(\nabla I_{h} G\left(u_{h}\right), \nabla \chi\right) & =\left(I_{h} f\left(u_{h}\right), \chi\right), \text { for } \chi \in S_{0 h}, 0<t<T, \\
u_{h}(0) & =v_{h} .
\end{aligned}
$$

Let $\left\{\phi_{i}\right\}_{i=1}^{M_{h}}$ be the nodal basis of $S_{h}$. Thus, the indices $1 \leqslant i \leqslant N_{h}$ refer to the interior nodes and the indices $N_{h}+1 \leqslant i \leqslant M_{h}$ refer to the boundary nodes. To compute $u_{h}$ from (1.3) one has to solve the system of ordinary differential equations

$$
\sum_{i=1}^{N_{h}} W_{i}^{\prime}\left(\phi_{i}, \phi_{j}\right)+\sum_{i=1}^{N_{h}} G\left(U_{i}\right)\left(\nabla \phi_{i}, \nabla \phi_{j}\right)=\sum_{i=1}^{M_{h}} f\left(U_{i}\right)\left(\phi_{i}, \phi_{j}\right), \quad j=1, \ldots, N_{h},
$$

where $U_{i}$ are the nodal values of $u_{h}$ and $W_{i}=H\left(U_{i}\right)$, subject to the initial conditions

$$
W_{j}(0)=H\left(V_{j}\right), j=1, \ldots, N_{h} .
$$

Here we use the fact that, assuming the coefficient $c$ to be positive, the enthalpy is a strictly increasing function, so that $U_{i}=H^{-1}\left(W_{i}\right)$ is uniquely defined. Thus, one actually computes an approximate enthalpy $w_{h}(t)=\Sigma_{i=1}^{N_{h}} W_{i}(t) \phi_{i} \in S_{0 h}$, from which the temperature $u_{h}(t)=\sum_{i=1}^{N_{h}} H^{-1}\left(W_{i}(t)\right) \phi_{i} \in S_{0 h}$ can be retrieved. Clearly, one can compute the standard mass and stiffness matrices $\left(\phi_{i}, \phi_{j}\right)$ and $\left(\nabla \phi_{i}, \nabla \phi_{j}\right)$ once and for all 
and then solve this system iteratively by some standard time-stepping procedure. We shall refrain from analysing this aspect of the problem.

In Section 4 below we estimate the $L_{2}$ and $H^{1}$ norms of the error in the approximate solution $u_{h}$ given by (1.3). We first show, for $r \geqslant 3, d \leqslant 3$, the error estimate

$$
\left\|u_{h}(t)-u(t)\right\|+\left(\int_{0}^{t}\left\|\nabla\left(u_{h}(\tau)-u(\tau)\right)\right\|^{2} \mathrm{~d} \tau\right)^{1 / 2} \leqslant C h^{r-1}, \text { for } 0 \leqslant t \leqslant T,
$$

where $\|\cdot\|$ denotes the norm in $L_{2}(\Omega)$. Note that the mean square average of the gradient of the error is of optimal order, whereas we have only been able to show a suboptimal error estimate pointwise in time. For the special case where $c \equiv 1$ we obtain, for $r \geqslant 3, d \leqslant 3$, a similar result, where again the $L_{2}$ norm of the error is one order less than optimal, pointwise in time, but where now the mean square average of the error is shown to be of optimal order $O\left(h^{r}\right)$. The case $r=2, d=1$ is somewhat particular and we obtain for general $c=c(u)$ an $O\left(h^{2}\right)$ error bound, pointwise in time.

The difficulty in this analysis stems from the way the interpolation is carried out under the gradient in the second term on the left-hand side. We therefore consider also the following method, where the coefficient $a\left(u_{h}\right)$ is interpolated directly: find $u_{h}$ : $[0, \mathrm{~T}] \rightarrow \mathrm{S}_{\mathrm{oh}}$ such that

$$
\begin{aligned}
\left(I_{h} H\left(u_{h}\right)_{t}, \chi\right)+\left(\left(I_{h} a\left(u_{h}\right)\right) \nabla u_{h}, \nabla \chi\right) & =\left(I_{h} f\left(u_{h}\right), \chi\right), \quad \text { for } \chi \in S_{0 h}, 0<t<T, \\
u_{h}(0) & =v_{h} .
\end{aligned}
$$

In matrix form this reads

$$
\begin{gathered}
\sum_{i=1}^{N_{h}} W_{i}^{\prime}\left(\phi_{i}, \phi_{j}\right)+\sum_{k=1}^{M_{h}} \sum_{i=1}^{N_{h}} a\left(U_{k}\right) U_{i}\left(\phi_{k} \nabla \phi_{i}, \nabla \phi_{j}\right)=\sum_{i=1}^{M_{h}} f\left(U_{i}\right)\left(\phi_{i}, \phi_{j}\right), \\
j=1, \ldots, N_{h}, \\
W_{j}(0)=H\left(V_{j}\right), \quad j=1, \ldots, N_{h},
\end{gathered}
$$

and the above remark about solvability applies to this system as well. We analyse this method in Section 3 and find that, provided that the initial approximation $v_{h}$ is chosen as an elliptic projection of $v$, the $L_{2}$ norm of the error is of optimal order pointwise in time for $r \geqslant 2$. For the special case, where $c(u) \equiv 1$, we show an optimal order error estimate without this restriction on $v_{h}$.

Several authors have considered numerical quadrature in finite element methods. The effect of quadrature in linear parabolic problems was analysed by Raviart. ${ }^{10}$ Christie et al. ${ }^{3}$ coined the term product approximation to refer to finite element techniques based on interpolation. Douglas and Dupont ${ }^{3}$ studied approximate problems of the type (1.4) with $c(u) \equiv 1$ and $f(u) \equiv 0$. In their work $I_{h}$ is allowed to be a more general projection. Nie and Thomée ${ }^{9}$, again with $c(u) \equiv 1$, considered the middle term in (1.4) in conjunction with the lumped mass method for the first term in a piecewise linear, two-dimensional setting. $\mathrm{Khalsa}^{8}$ analysed a finite element method with product approximation for a semilinear parabolic problem with a cubic nonlinearity in one space dimension.

The present work was inspired by the papers of Cermák and Zlámal ${ }^{2}$ and Borshukova and Konovski, ${ }^{1}$ in which the method (1.3) was applied to various heat conduction problems with and without phase change. These papers report on numerical computations and contain no error analysis. Our analysis does not allow phase change, i.e. we do not allow $H(u)$ and $G(u)$ to be non-smooth functions of $u$. 
Such a problem was, however, analysed by Elliott. ${ }^{6} \mathrm{He}$ assumed that $H(u)$ has a jump discontinuity and that $G(u)=u$ and $f=f(x, t)$. For a completely discrete version of $(1.3)$, using a piecewise linear finite element method for the spatial discretization, he obtained an $O\left(h^{1 / 2}\right)$ estimate for the mean square average in time of the $L_{2}$ norm of the error.

The product approximation for semilinear elliptic problems was analysed by SanzSerna and Abia. ${ }^{11}$ Their analysis is based on inverse inequalities and a continuation argument, an approach that we have adopted here, too.

\section{Notation and preliminaries}

In this section we state our general assumptions about the non-linear initialboundary value problem (1.1) and the finite element methods to be analysed. We also collect some notation and preliminary results.

Let $\Omega$ be a bounded polygonal domain in $\mathbb{R}^{d}$ with $d \leqslant 3$. We shall assume that the coefficients $c(u), a(u)$ and $f(u)$ of (1.1) are smooth functions of $u \in \mathbb{R}$ and that $c$ and $a$ are uniformly positive:

$$
c(u) \geqslant c_{0}>0, a(u) \geqslant a_{0}>0 \text { for all } u \in \mathbb{R} .
$$

We further assume that (1.1) has a unique solution $u$, which is sufficiently smooth for our purposes. Throughout this paper we thus make the somewhat unrealistic-but commonplace-assumption that the solution of problem (1.1) is very smooth, in spite of the polygonal character of the domain $\Omega$.

For the approximation of (1.1) we shall consider standard piecewise polynomial Lagrangian finite element spaces. Thus, we assume that we have a quasi-uniform family $\left\{\tau_{h}\right\}_{h>0}$ of simplicial triangulations of $\Omega$ with the parameter $h$ being the maximal diameter of any simplex $K$ in $\tau_{h}$. Further, for some integer $r \geqslant 2$, we denote by $S_{h}$ the space of continuous functions that reduce to polynomials of degree $\leqslant r-1$ on each simplex $K \in \tau_{h}$, and we let $S_{0 h}=\left\{\chi \in S_{h}:\left.\chi\right|_{o \Omega}=0\right\}$. Thus, we have $S_{h} \subset H^{1}(\Omega)$ and $S_{0 k} \subset H_{0}^{1}(\Omega)$.

We shall use the notation $(\cdot, \cdot)$ and $\|\cdot\|$ for the inner product and norm of $L_{2}=L_{2}(\Omega)$ and $\|\cdot\|_{m, p}$ for the norms of the Sobolev spaces $W_{p}^{m}=W_{p}^{m}(\Omega)$. For $p=2$ we write $H^{m}=H^{m}(\Omega)$ and $\|\cdot\|_{m}$. These norms should be interpreted in the piecewise sense, when applied to functions that are only piecewise differentiable with respect to $\tau_{k}$. Further, we write

$$
\|v\|_{L,(0, r ; X)}=\left(\int_{0}^{t}\|v(\tau)\|_{X}^{p} \mathrm{~d} \tau\right)^{1 / p},
$$

with the usual modification for $p=\infty$ and where $X$ could be any of the Banach spaces mentioned above.

We define the interpolation operator $I_{h}: C(\bar{\Omega}) \rightarrow S_{h}$ by the condition that $\left(I_{h} v\right)(P)=$ $v(P)$ for any of the nodes $P$ that define the degrees of freedom of $S_{k}$. From the theory of finite elements we quote the following error estimate: for $0 \leqslant m \leqslant r$ and $1 \leqslant p \leqslant \infty$ we have

$$
\left\|I_{h} v-v\right\|_{m, p} \leqslant C h^{r-m}\|v\|_{r, p},
$$


if $v$ belongs to $C(\bar{\Omega})$ and $W_{p}^{r}(K)$ for all $K \in \tau_{h}$, (see, for instance, Reference 4, Theorem 3.1.6).

We shall often need to be able to estimate high order norms of the error in terms of lower order norms. This can be done by an inverse inequality argument, which we state in the following lemma.

Lemma 1. Let $0 \leqslant l \leqslant m \leqslant r, 1 \leqslant q \leqslant p \leqslant \infty$. Then for $\chi \in S_{h}$ and $v \in W_{p}^{r}$ we have

$$
\|\chi-v\|_{m, p} \leqslant C h^{-(m-l)-[(d / q)-(d / p)]}\left(\|\chi-v\|_{l, q}+h^{r-l}\|v\|_{r, p}\right) \text {. }
$$

Proof. Using (2.2) and an inverse inequality (Reference 4, Theorem 3.2.6) we obtain

$$
\begin{aligned}
\|\chi-v\|_{m, p} \leqslant & \left\|\chi-I_{h} v\right\|_{m, p}+\left\|I_{h} v-v\right\|_{m, p} \\
\leqslant & C h^{-(m-l)-[(d / q)-(d / p)]}\left\|\chi-I_{h} v\right\|_{l, q}+\left\|I_{h} v-v\right\|_{m, p} \\
\leqslant & C h^{-(m-l)-[(d / q)-(d / p)]}\left(\|\chi-v\|_{l, q}+\left\|I_{h} v-v\right\|_{l, q}\right) \\
& +\left\|I_{h} v-v\right\|_{m, p} \\
\leqslant & C h^{-(m-l)-[(d / q)-(d / p)]}\left(\|\chi-v\|_{l, q}+C h^{r-1}\|v\|_{r, q}\right) \\
& +C h^{r-m}\|v\|_{r, p},
\end{aligned}
$$

which proves the lemma.

In our error analysis we shall also use a Ritz projection $\tilde{u}_{h}=\tilde{u}_{h}(t) \in S_{0 h}$ of the exact solution $u$ of (1.1). For fixed $t \in[0, T]$, we define this to be the solution of the linear problem

$$
\left(a(u(t)) \nabla\left(\tilde{u}_{h}(t)-u(t)\right), \nabla \chi\right)=0, \text { for all } \chi \in S_{0 h} .
$$

To discuss this definition (and for later reference) we consider the linear elliptic problem

$$
\begin{aligned}
\nabla \cdot(a(u) \nabla w)=g, & \text { in } \Omega \times(0, T), \\
w=0, & \text { on } \partial \Omega \times(0, T) .
\end{aligned}
$$

We may define the solution operator $T=T(u(t)): L_{2}(\Omega) \rightarrow H_{0}^{1}(\Omega) \cap H^{2}(\Omega)$ (not to be mistaken for the length of the time interval) by

$$
(a(u) \nabla T g, \nabla \chi)=(g, \chi), \quad \chi \in H_{0}^{1}(\Omega) .
$$

The corresponding approximate solution operator $T_{h}=T_{h}(u(t)): L_{2}(\Omega) \rightarrow S_{0 h}$ is given by

$$
\left(a(u) \nabla T_{h} g, \nabla \chi\right)=(g, \chi), \quad \chi \in S_{0 h} .
$$

In order to be able to perform the duality argument of standard error analysis, we need to assume that $\Omega$ is such that

$$
\|T g\|_{2} \leqslant C\|g\|, \quad g \in L_{2}(\Omega),
$$

so that

$$
\left\|\left(T_{h}-T\right) g\right\|_{1} \leqslant C h\|g\|, \quad g \in L_{2}(\Omega),
$$

It is well known that this holds, for instance, for convex polygonal domains (see Theorem 3.2.1.2 of Reference 7 and its proof). 
In our next lemma we collect some error estimates and maximum norm bounds for $\tilde{u}_{k}$.

Lemn 2. There is a constant $C=C(u)$ such that for $0 \leqslant t \leqslant T$ we have

and

$$
\left\|D_{z}^{j}\left(\tilde{u}_{h}-u\right)(t)\right\|_{l} \leqslant C h^{r-1}, \text { for } j, l=0,1,
$$

$$
\left\|D_{t}^{j} \tilde{u}_{h}(t)\right\|_{1, \infty} \leqslant C, \text { for } j=0,1 .
$$

Proof. With the Ritz projection operator $R_{h}=R_{h}(u(t)): H_{0}^{1}(\Omega) \rightarrow S_{0 h}$ defined by

$$
\left(a(u(t)) \nabla\left(R_{h} w-w\right), \nabla \chi\right)=0, \text { for all } \chi \in S_{0 h},
$$

we have $\tilde{u}_{h}=R_{h} u$. The case $j=0$ of (2.8) now follows from the standard error analysis for linear elliptic problems with variable coefficients. The case $j=1$ follows in a straightforward way after differentiation of (2.4) with respect to time,

$$
\left(a \nabla\left(\tilde{u}_{h, t}-u_{t}\right), \nabla \chi\right)+\left(a_{t} \nabla\left(\tilde{u}_{h}-u\right), \nabla \chi\right)=0, \text { for all } \chi \in S_{0 h},
$$

where we have written $a$ for $a(u)$ and $a_{t}$ for $a(u)_{t}=a^{\prime}(u) u_{t}$. We refer to Reference 13 (Lemmas 2 and 3 in Chapter 5) for the details.

For the proof of (2.9) we shall use the maximum norm stability of $R_{h}$,

$$
\left\|R_{h} w\right\|_{0, \infty} \leqslant C(\log (1 / h))^{r}\|w\|_{0, \infty},
$$

where $\bar{r}=1$ if $r=2, \bar{r}=0$ otherwise, (cf. (5.9)' in Reference 12). (Although the results of Reference 12 are formulated for a model problem with constant coefficients, the authors remark that their methods work in our more general situation, as well.) Let $\rho=\tilde{u}_{h}-u$. An application of (2.12) with $w=u(t)-\chi, \chi \in S_{0 h}$ arbitrary, shows

$$
\|\rho(t)\|_{0, \infty} \leqslant C h^{\prime}(\log (1 / h))^{\bar{r}},
$$

so that, by Lemma $1,\|\rho(t)\|_{1, \infty} \leqslant C$ and the case $j=0$ of (2.9) follows.

Next we note that (2.11) can be written

$$
\left(a \nabla\left(\tilde{u}_{h, t}-u_{t}\right), \nabla \chi\right)+\left(a \nabla\left(\frac{a_{t}}{a} \rho\right), \nabla \chi\right)-\left(a \rho \nabla\left(\frac{a_{t}}{a}\right), \nabla \chi\right)=0 .
$$

This means that $\tilde{u}_{h, t}-R_{h} u_{t}+R_{h}\left(\left(a_{t} / a\right) \rho\right)=\eta$ is an element of $S_{0 h}$, which satisfies

$$
(a \nabla \eta, \nabla \chi)=\left(a \rho \nabla\left(\frac{a_{t}}{a}\right), \nabla \chi\right) \text {, for all } \chi \in S_{0 h} \text {. }
$$

By the same token as for $\tilde{u}_{h}=R_{h} u$, we have $\left\|R_{h} u_{l}(t)\right\|_{1, \infty} \leqslant C$. By (2.12) and (2.13), we next obtain

$$
\left\|R_{h}\left(\frac{a_{t}}{a} \rho\right)(t)\right\|_{0, \infty} \leqslant C(\log (1 / h))^{\|}\left\|\frac{a_{t}}{a} \rho(t)\right\|_{0, \infty} \leqslant C h^{r}(\log (1 / h))^{2 r},
$$

which implies $\left\|R_{h}\left(\left(a_{t} / a\right) \rho\right)(t)\right\|_{1, \infty} \leqslant C$ in view of an inverse inequality.

Finally, setting $\chi=\eta$ in (2.14), we obtain

$$
\|\eta(t)\|_{1} \leqslant C\left\|a \rho \nabla\left(\frac{a_{i}}{a}\right)\right\| \leqslant C\|\rho(t)\|
$$


and an inverse inequality and (2.8) show

$$
\|\eta(t)\|_{1, \infty} \leqslant C h^{r-d / 2} \leqslant C .
$$

Since $\tilde{u}_{h, t}=R_{h} u_{t}-R_{h}\left(\left(a_{t} / a\right) \rho\right)+\eta$, this proves the remaining case $j=1$ of $(2.9)$ and the proof of the lemma is complete.

\section{Analysis of the second method}

In this section we shall analyse the following interpolated coefficient finite element method for the non-linear heat equation (1.1): find $u_{h}:[0, T] \rightarrow S_{0 h}$ such that

$$
\begin{aligned}
\left(I_{h} H\left(u_{h}\right)_{t}, \chi\right)+\left(\left(I_{h} a\left(u_{h}\right)\right) \nabla u_{h}, \nabla \chi\right) & =\left(I_{h} f\left(u_{h}\right), \chi\right), \text { for } \chi \in S_{0 h}, 0<t<T, \\
u_{h}(0) & =v_{h} .
\end{aligned}
$$

We shall show an error estimate, which is of optimal order, provided that the initial approximation is chosen as the Ritz projection $\tilde{v}_{h}=\tilde{u}_{h}(0)$ of $v$ defined in Section 2.

Theorem 1. Let $u_{h}$ and $u$ be the solutions of (3.1) and (1.1), respectively, and assume that $v_{h}=\tilde{v}_{h}=\tilde{u}_{h}(0)$. Then there are positive numbers $h_{0}=h_{0}(u, T)$ and $C=C(u, T)$ such that, for $h<h_{0}$, we have

$$
\left\|u_{h}(t)-u(t)\right\|+h\left\|u_{h}(t)-u(t)\right\|_{1} \leqslant C h^{r}, \text { for } 0 \leqslant t \leqslant T .
$$

In the proof of Theorem 1 we shall follow the standard method of splitting the error into two parts,

$$
e=u_{h}-u=\left(u_{h}-\tilde{u}_{h}\right)+\left(\tilde{u}_{h}-u\right)
$$

where $\tilde{u}_{h}$ is the Ritz projection of $u$ defined in Section 2. In Lemma 2 we found that $\tilde{u}_{h}$ $-u$ satisfies the desired error estimate and so it remains to estimate $\theta=u_{h}-\tilde{u}_{h}$. This will be done in the following lemma. The proof of the theorem will then be completed by means of a continuation argument.

Lemma 3. In addition to the assumptions of Theorem 1, assume that, for some $t_{1}$ with $0<t_{1} \leqslant T$, we have

$$
\|e\|_{L_{\infty}\left(0, t_{1} ; L_{2}\right)}+\left\|e_{t}\right\|_{L_{2}\left(0, t_{1} ; L_{2}\right)} \leqslant h^{r-1 / 4} \text {. }
$$

Then it follows that

$$
\|e\|_{L_{\infty}\left(0, t_{1} ; L_{2}\right)}+h\|e\|_{L_{\infty}\left(0, t_{1} ; H^{1}\right)}+\left\|e_{t}\right\|_{L_{2}\left(0, t_{1} ; L_{2}\right)} \leqslant C h^{r},
$$

where $C=C(u, T)$ does not depend on $t_{1}$.

Proof. Let us first note that, by the inverse inequality argument of Lemma 1 , and since $r \geqslant 2$ and $d \leqslant 3$, the hypothesis (3.2) implies

$$
\|e\|_{L_{\infty}\left(0, t_{1} ; w_{4}^{-1}\right)} \leqslant C, \quad\|e\|_{L_{\infty}\left(0, t_{1} ; w_{\infty}^{r-2}\right)} \leqslant C
$$

and

$$
\left\|e_{t}\right\|_{L_{2}\left(0, t_{1} ; W_{4}^{r-1}\right)} \leqslant C, \quad\left\|e_{t}\right\|_{L_{2}\left(0, t_{1} ; W_{\infty}^{r-2}\right)} \leqslant C,
$$

so that, in particular, since $u$ is smooth,

$$
\left\|u_{h}\right\|_{L_{\infty}\left(0, t_{1} ; W_{4}^{r-1}\right)} \leqslant C, \quad\left\|u_{h}\right\|_{L_{\infty}\left(0, t_{1} ; W_{\infty}^{r-2}\right)} \leqslant C
$$


and

$$
\left\|u_{h, t}\right\|_{L_{2}\left(0, t_{1} ; W^{-1}\right)} \leqslant C, \quad\left\|u_{h, s}\right\|_{L_{2}\left(0, t_{1} ; W_{\infty}^{\prime-2}\right)} \leqslant C .
$$

Using these bounds we shall next show that

$$
\begin{aligned}
& \left\|f\left(u_{h}\right)\right\|_{L_{\infty}\left(0, r_{1} ; H^{\prime}\right)} \leqslant C, \\
& \left\|a\left(u_{h}\right)\right\|_{L_{\infty}\left(0, t_{1} ; H^{\prime}\right)} \leqslant C, \\
& \left\|H\left(u_{h}\right)_{t}\right\|_{L_{2}\left(0, t_{1} ; H^{\prime}\right)} \leqslant C, \\
& \left\|a\left(u_{h}\right)_{l}\right\|_{L_{2}\left(0, t_{1} ; H^{\prime}\right)} \leqslant C .
\end{aligned}
$$

For the proof of (3.5) we let $|\alpha|=r$ and apply Hölder's inequality to the formula

$$
D^{\alpha} f\left(u_{h}\right)=\sum_{i=1}^{r} \sum_{\substack{\Sigma_{i}^{i} \beta_{j}=\alpha \\ \beta_{j} \neq 0}} f^{(i)}\left(u_{h}\right) D^{\beta_{1}} u_{h} \ldots D^{\beta_{i}} u_{h},
$$

which, since $\left\|u_{h}\right\|_{L_{\infty}\left(0, t_{1} ; L_{\infty}\right)} \leqslant C$ by (3.3), implies

$$
\left\|D^{\alpha} f\left(u_{h}\right)\right\| \leqslant C \sum_{\substack{\Sigma_{i}, \beta_{j}=\alpha \\ \beta_{j} \neq 0}}\left\|D^{\beta_{1}} u_{h}\right\|_{0, q_{1}} \ldots\left\|D^{\beta_{i}} u_{h}\right\|_{0, q_{1}},
$$

where $\Sigma_{1}^{i} 1 / q_{j}=1 / 2$. Now note that -elementwise-any $r$ th order derivative of $u_{h}$ is identically zero and that factors $D^{\beta} u_{h}$ with $\left|\beta_{j}\right|=r-1$ can occur at most twice in any of the products in equation (3.9)-in fact, they occur twice only if $r=2$. Thus, we may take $q_{j}=2$ or 4 if $\left|\beta_{j}\right|=r-1$ and $q_{j}=\infty$ otherwise and (3.5) follows in view of the bounds in (3.3).

For (3.7) we have in a similar. manner

$$
\begin{aligned}
D^{\alpha} H\left(u_{h}\right)_{l} & =\sum_{\beta_{0}+\beta=\alpha} D^{\beta_{0}} u_{h, t} D^{\beta} c\left(u_{h}\right) \\
& =\sum_{\beta_{0}+\beta=\alpha} \sum_{i=0}^{|\beta|} D^{\beta 0} u_{h, t} \sum_{\substack{\Sigma_{1} \\
\beta_{j} \neq \beta \\
\beta, 0}} c^{(i)}\left(u_{h}\right) D^{\beta_{1}} u_{h} \ldots D^{\beta_{i}} u_{h},
\end{aligned}
$$

so that

$$
\begin{aligned}
\left\|D^{\alpha} H\left(u_{h}\right)_{t}\right\|_{L_{2}\left(0, t_{1} ; L_{2}\right) \leqslant} & C \sum_{\Sigma_{0}^{1} \beta_{j}=\alpha}\left\|D^{\beta_{0}} u_{h, t}\right\|_{L_{2}\left(0, t_{1} ; L_{\infty 0}\right)} \\
& \times\left\|D^{\beta_{1}} u_{h}\right\|_{L_{\infty}\left(0, t_{1} ; L_{q_{1}}\right)} \ldots\left\|D^{\beta_{i}} u_{h}\right\|_{L_{\infty}\left(0, t_{1} ; L_{q}\right)},
\end{aligned}
$$

where $\Sigma_{0}^{i} 1 / q_{j}=1 / 2$ and (3.7) follows by the same argument as above. The bounds (3.6) and (3.8) are proved in the same way as (3.5) and (3.7).

Next we shall bound $\theta=u_{h}-\tilde{u}_{h}$. Consider first the case $r>2$. Using (3.1), (2.4) and the weak form of equation (1.1), we have, for $\chi \in S_{0 h}$ and $0<t<t_{1}$,

$$
\begin{aligned}
& \left(c\left(u_{h}\right) \theta_{t}, \chi\right)+\left(a\left(u_{h}\right) \nabla \theta, \nabla \chi\right)=\left(\left(I_{h}-I\right) f\left(u_{h}\right), \chi\right)+\left(f\left(u_{h}\right)-f(u), \chi\right) \\
& -\left(\left(I_{h}-I\right) H\left(u_{h}\right)_{h}, \chi\right)-\left(\left(c\left(u_{h}\right)-c(u)\right) u_{t}, \chi\right)-\left(c\left(u_{h}\right)\left(\tilde{u}_{k, t}-u_{t}\right), \chi\right) \\
& -\left(\left(\left(I_{h}-I\right) a\left(u_{h}\right)\right) \nabla u_{h}, \nabla \chi\right)-\left(\left(a\left(u_{h}\right)-a(u)\right) \nabla \tilde{u}_{h}, \nabla \chi\right) \\
& =\sum_{i=1}^{S}\left(R_{i}, \chi\right)+\sum_{i=6}^{7}\left(R_{i}, \nabla \chi\right),
\end{aligned}
$$


where $l$ denotes the identity operator and with the obvious definitions of the terms $\boldsymbol{R}_{\boldsymbol{i}}$. Taking $\chi=\theta_{t}$, we obtain

$$
\begin{aligned}
\left(c\left(u_{h}\right) \theta_{i}, \theta_{t}\right)+\frac{1 \mathrm{~d}}{2 \mathrm{~d} t}\left(a\left(u_{h}\right) \nabla \theta, \nabla \theta\right)= & \sum_{i=1}^{5}\left(R_{i}, \theta_{i}\right)+\frac{\mathrm{d}}{\mathrm{d} t} \sum_{i=6}^{7}\left(R_{i}, \nabla \theta\right) \\
& -\sum_{i=6}^{7}\left(R_{i, t}, \nabla \theta\right)+\left(R_{8}, \nabla \theta\right),
\end{aligned}
$$

where $R_{8}=1 / 2 a\left(u_{h}\right)_{t} \nabla \theta$. Integration with respect to $t$, using (2.1) and the fact that $\theta(0)=0$, yields

$$
\begin{aligned}
\int_{0}^{t}\left\|\theta_{t}\right\|^{2} \mathrm{~d} \tau+\|\theta(t)\|_{1}^{2} \leqslant C & \sum_{i=1}^{5} \int_{0}^{t}\left\|R_{i}\right\|\left\|\theta_{t}\right\| \mathrm{d} \tau+C \sum_{i=6}^{7}\left\|R_{i}(t)\right\|\|\theta(t)\|_{1} \\
& +C \int_{0}^{t}\left(\left\|R_{6, t}\right\|+\left\|R_{7, t}\right\|+\left\|R_{8}\right\|\right)\|\theta\|_{1} \mathrm{~d} \tau,
\end{aligned}
$$

so that, after trivial estimates and a simple kick-back argument,

$$
\begin{aligned}
\left\|\theta_{i}\right\|_{L_{2}\left(0, i ; L_{2}\right)}^{2}+\|\theta\|_{L_{\infty}\left(0, r_{i} H^{1}\right)}^{2} \leqslant & C \sum_{i=1}^{5}\left\|R_{i}\right\|_{L_{2}\left(0, i ; L_{2}\right)}^{2}+C \sum_{i=6}^{7}\left\|R_{i}\right\|_{L_{\infty}\left(0, t: L_{2}\right)}^{2} \\
& +C \int_{0}^{t}\left(\left\|R_{6, i}\right\|+\left\|R_{7,1}\right\|+\left\|R_{8}\right\|\right)\|\theta\|_{1} \mathrm{~d} \tau .
\end{aligned}
$$

Here we have to bound the various terms on the right-hand side. To begin with, using (2.2), (3.5) and (3.7), we have

$$
\begin{aligned}
& \left\|R_{1}\right\|_{L_{2}\left(0, t ; L_{2}\right)} \leqslant C h^{r}\left\|f\left(u_{h}\right)\right\|_{L_{2}\left(0, r, H^{\prime}\right)} \leqslant C h^{r}, \\
& \left\|R_{3}\right\|_{L_{2}\left(0, r, L_{2}\right)} \leqslant C h^{r}\left\|H\left(u_{h}\right)_{1}\right\|_{L_{2}\left(0, r ; H^{\prime}\right)} \leqslant C h^{r} .
\end{aligned}
$$

Next, since $u_{h}$ is bounded by (3.3), using (2.8) we obtain

$$
\begin{aligned}
\left\|R_{2}\right\|_{L_{2}\left(0, t ; L_{2}\right)}+\left\|R_{4}\right\|_{L_{2}\left(0, r, L_{2}\right)} & \leqslant C\left\|u_{h}-u\right\|_{L_{2}\left(0, r ; L_{2}\right)} \\
& \leqslant C\left(\left\|\tilde{u}_{h}-u\right\|_{L_{2}\left(0, r ; L_{2}\right)}+\|\theta\|_{L_{2}\left(0, r ; L_{2}\right)}\right) \\
& \leqslant C h^{r}+C\|\theta\|_{L_{2}\left(0, r ; H^{\prime}\right)},
\end{aligned}
$$

and

$$
\left\|R_{5}\right\|_{L_{2}\left(0, t ; L_{2}\right)} \leqslant C\left\|\tilde{u}_{h, t}-u_{t}\right\|_{L_{2}\left(0, t ; L_{2}\right)} \leqslant C h^{r} .
$$

To obtain bounds of $R_{6}$ and $R_{6, t}$ we note that, by (3.3) and (3.4) and since $r \geqslant 3, u_{h}$ and $u_{h, t}$ are bounded in $L_{\infty}\left(0, t ; W_{\infty}^{1}\right)$ and $L_{2}\left(0, t ; W_{\infty}^{1}\right)$, respectively. We find

$$
\begin{aligned}
\left\|R_{6}\right\|_{L_{\infty}\left(0, t ; L_{2}\right)} & \leqslant\left\|\left(I_{h}-I\right) a\left(u_{h}\right)\right\|_{L_{\infty}\left(0, r ; L_{2}\right)}\left\|\nabla u_{h}\right\|_{L_{\infty}\left(0, r ; L_{\infty}\right)} \\
& \leqslant C h^{r}\left\|a\left(u_{h}\right)\right\|_{L_{\infty}\left(0, r ; H^{r}\right)}\left\|u_{h}\right\|_{L_{\infty}\left(0, r ; W_{\infty}^{1}\right)} \leqslant C h^{r},
\end{aligned}
$$


by (3.6), and

$$
\begin{aligned}
\int_{0}^{t}\left\|R_{6, t}\right\|\|\theta\|_{1} \mathrm{~d} \tau \leqslant & \left\|R_{6, t}\right\|_{L_{2}\left(0, t, L_{2}\right)}\|\theta\|_{L_{2}\left(0, t ; H^{1}\right)} \\
\leqslant & \left(\left\|\left(I_{h}-I\right) a\left(u_{h}\right)\right\|_{L_{2}\left(0, t ; L_{2}\right)}\left\|\nabla u_{h}\right\|_{L_{\infty}\left(0, r, L_{\infty}\right)}\right. \\
& \left.+\left\|\left(I_{h}-I\right) a\left(u_{h}\right)\right\|_{L_{\infty}\left(0, t ; L_{2}\right)}\left\|\nabla u_{h_{1}, t}\right\|_{L_{2}\left(0, t ; L_{\infty}\right)}\right)\|\theta\|_{L_{2}\left(0, r, H^{1}\right)} \\
\leqslant & C h^{r}\left(\left\|a\left(u_{h}\right)_{t}\right\|_{L_{2}\left(0, t ; H^{r}\right)}\left\|u_{h}\right\|_{L_{\infty}\left(0, t, W_{\infty}^{1}\right)}\right. \\
& \left.+\left\|a\left(u_{h}\right)\right\|_{L_{\infty}\left(0, t ; H^{r}\right)}\left\|u_{h, t}\right\|_{L_{2}\left(0, t ; W_{\infty}^{2}\right)}\right)\|\theta\|_{L_{2}\left(0, t ; H^{1}\right)} \\
\leqslant & C h^{r}\|\theta\|_{L_{2}\left(0, t, H^{1}\right)} \leqslant C h^{2 r}+C\|\theta\|_{L_{2}\left(0, t, H^{1}\right)}^{2},
\end{aligned}
$$

by (3.8) and (3.6). To estimate $R_{7}$ and $R_{7, t}$ we shall use the bounds of $\tilde{u}_{h}$ and $\tilde{u}_{h, t}$ in (2.9). First we obtain

$$
\begin{aligned}
\left\|R_{7}\right\|_{L_{\infty}\left(0, r ; L_{2}\right)} & \leqslant\left\|a\left(u_{h}\right)-a(u)\right\|_{L_{\infty}\left(0, r ; L_{2}\right)}\left\|\nabla \tilde{u}_{h}\right\|_{L_{\infty}\left(0, r ; L_{\infty}\right)} \\
& \leqslant C\left\|u_{h}-u\right\|_{L_{\infty}\left(0, t ; L_{2}\right)} \leqslant C\left(\left\|\tilde{u}_{h}-u\right\|_{L_{\infty}\left(0, r, L_{2}\right)}+\|\theta\|_{L_{\infty}\left(0, t ; L_{2}\right)}\right) \\
& \leqslant C h^{r}+C\|\theta\|_{L_{\infty}\left(0, t ; L_{2}\right)}
\end{aligned}
$$

where, for some $\tilde{t} \in[0, t]$,

$$
\begin{aligned}
\|\theta\|_{L_{\infty}\left(0, t ; L_{2}\right)}^{2} & =\|\theta(\bar{t})\|^{2}=\int_{0}^{\tau} \frac{\mathrm{d}}{\mathrm{d} \tau}\|\theta\|^{2} \mathrm{~d} \tau \leqslant 2 \int_{0}^{t}\|\theta\|\left\|\theta_{t}\right\| \mathrm{d} \tau \\
& \leqslant 2\|\theta\|_{L_{2}\left(0, r ; L_{2}\right)}\left\|\theta_{t}\right\|_{L_{2}\left(0, r_{i} L_{2}\right)},
\end{aligned}
$$

since $\theta(0)=0$. Thus, for any $\varepsilon>0$, we can select $C$ such that

$$
\left\|R_{7}\right\|_{L_{\infty}\left(0, t ; L_{2}\right)}^{2} \leqslant C h^{2 r}+C\|\theta\|_{L_{2}\left(0, t ; H^{\prime}\right)}^{2}+\varepsilon\left\|\theta_{t}\right\|_{L_{2}\left(0, t ; L_{2}\right)}^{2}
$$

Similarly,

$$
\begin{aligned}
\int_{0}^{t}\left\|R_{7, t}\right\|\|\theta\|_{1} \mathrm{~d} \tau \leqslant & \left\|R_{7, t}\right\|_{L_{2}\left(0, t ; L_{2}\right)}\|\theta\|_{L_{2}\left(0, t ; H^{\prime}\right)} \\
\leqslant & \left(\left\|\left(a\left(u_{h}\right)-a(u)\right)_{t}\right\|_{L_{2}\left(0, t ; L_{2}\right)}\left\|\nabla \tilde{u}_{h}\right\|_{L_{\infty}\left(0, t ; L_{\infty}\right)}\right. \\
& \left.+\left\|a\left(u_{h}\right)-a(u)\right\|_{L_{2}\left({ }^{\prime} t ; L_{2}\right)}\left\|\nabla \tilde{u}_{h_{t} t}\right\|_{L_{\infty}\left(0, t ; L_{\infty}\right)}\right)\|\theta\|_{L_{2}\left(0, t ; H^{\prime}\right)} \\
\leqslant & C\left(\left\|u_{h}-u\right\|_{L_{2}\left(0, t ; L_{2}\right)}+\left\|u_{h, t}-u_{t}\right\|_{L_{2}\left(0, t ; L_{2}\right)}\right)\|\theta\|_{L_{2}\left(0, t ; H^{1}\right)} \\
\leqslant & C\left(\|\theta\|_{L_{2}\left(0, t ; L_{2}\right)}+\left\|\tilde{u}_{h}-u\right\|_{L_{2}\left(0, t ; L_{2}\right)}\right. \\
& \left.+\|\theta\|_{L_{2}\left(0, t ; L_{2}\right)}+\left\|\tilde{u}_{h, t}-u_{t}\right\|_{L_{2}\left(0, t ; L_{2}\right)}\right)\|\theta\|_{L_{2}\left(0, t ; H^{1}\right)} \\
\leqslant & C h^{2 r}+C\|\theta\|_{L_{2}\left(0, t ; H^{1}\right)}^{2}+\varepsilon\left\|\theta_{t}\right\|_{L_{2}\left(0, t ; L_{2}\right)}^{2} .
\end{aligned}
$$

Finally, from (3.4) it follows that

$$
\begin{aligned}
\int_{0}^{t}\left\|R_{8}\right\|\|\theta\|_{1} \mathrm{~d} \tau & \leqslant C\left\|u_{h, 1}\right\|_{L_{2}\left(0, t ; L_{\infty}\right)}\|\theta\|_{L_{\infty 0}\left(0, r_{;} H^{1}\right)}\|\theta\|_{L_{2}\left(0, t ; H^{1}\right)} \\
& \leqslant C\|\theta\|_{L_{2}\left(0, t ; H^{1}\right)}^{2}+\varepsilon\|\theta\|_{L_{\infty}\left(0, t ; H^{2}\right)}^{2}
\end{aligned}
$$


Summing up, choosing $\varepsilon$ appropriately, we now have

$$
\|\theta\|_{L_{\infty}\left(0, t ; H^{1}\right)}^{2}+\left\|\theta_{1}\right\|_{L_{2}\left(0, r ; L_{2}\right)}^{2} \leqslant C h^{2 r}+C\|\theta\|_{L_{2}\left(0, r, H^{1}\right)}^{2} \text {, for } 0<t<t_{1},
$$

which, by Grönwall's inequality, shows

$$
\|\theta\|_{L_{\infty}\left(0, t_{1} ; H^{1}\right)}^{2}+\left\|\theta_{t}\right\|_{L_{2}\left(0, t_{1} ; L_{2}\right)}^{2} \leqslant C h^{2 r} .
$$

Together with the appropriate estimates of $\tilde{u}_{h}-u$ from Lemma 2 this proves the case $r>2$ of the lemma.

If $r=2$ (and $d=3$ ), then (3.3) and (3.4) no longer imply $W_{\infty}^{1}$ bounds for $u_{h}$ and $u_{h, t}$. But, on the other hand, (2.1) now implies that $I_{h} a\left(u_{h}\right) \geqslant a_{0}>0$ and we shall use the following variant of equation (3.12):

$$
\begin{gathered}
\left(c\left(u_{h}\right) \theta_{t}, \chi\right)+\left(I_{h} a\left(u_{h}\right) \nabla \theta, \nabla \chi\right)=\left(\left(I_{h}-I\right) f\left(u_{h}\right), \chi\right)+\left(f\left(u_{h}\right)-f(u), \chi\right) \\
-\left(\left(I_{h}-I\right) H\left(u_{h}\right)_{t}, \chi\right)-\left(\left(c\left(u_{h}\right)-c(u)\right) u_{t}, \chi\right)-\left(c\left(u_{h}\right)\left(\tilde{u}_{h, t}-u_{t}\right), \chi\right) \\
-\left(\left(\left(I_{h}-I\right) a\left(u_{h}\right)\right) \nabla \tilde{u}_{h}, \nabla \chi\right)-\left(\left(a\left(u_{h}\right)-a(u)\right) \nabla \tilde{u}_{h}, \nabla \chi\right) .
\end{gathered}
$$

Thus, all terms involving $a\left(u_{h}\right)$ can now be estimated using the maximum norm bounds of $\tilde{u}_{h}$ and $\tilde{u}_{h, s}$ in Lemma 2 . This completes the proof.

Proof of Theorem 1. Let $t_{1}^{*}$ be the largest $t_{1}$ such that (3.2) holds. It is obvious that $t_{1}^{*}>0$. If $t_{1}^{*}<T$, then by Lemma 3 we can find $h_{0}>0$ such that, for $h<h_{0}$, we have

$$
\|e\|_{L_{\infty}\left(0, t_{1}^{*} ; L_{2}\right)}+\left\|e_{t}\right\|_{L_{2}\left(0, t_{1}^{*} ; L_{2}\right)} \leqslant C h^{r} \leqslant \frac{1}{2} h^{r-1 / 4},
$$

in contradiction to the maximality of $t_{1}^{*}$. Thus $t_{1}^{*}=T$ and the proof is complete.

In Theorem 1 we assumed that $v_{h}=\tilde{v}_{h}$ in order to be able to prove an $O\left(h^{r}\right)$ error estimate for $\left\|\theta_{t}\right\|_{L_{2}\left(0, t_{i} L_{2}\right)}$, which was needed because of the non-linearity in the coefficient $c(u)$-recall how the bound (3.7) was used in estimating the term $R_{3}$ in the proof of Lemma 3. (Clearly, it is sufficient to choose $v_{h}$ in such a way that $\left\|v_{h}-\tilde{v}_{h}\right\|_{1}$ $=\|\theta(0)\|_{1}$ is of superconvergent order $O\left(h^{r}\right)$.) In our next result we shall assume that $c$ does not depend on $u$-for simplicity we take $c(u) \equiv 1$ - and we shall prove an error estimate without any such restriction on $v_{h}$.

Theorem 2. Let $c(u) \equiv 1$ and let $u_{h}$ and $u$ be the solutions of (3.1) and (1.1), respectively, and assume that

$$
\left\|v_{h}-v\right\| \leqslant C h^{r}
$$

Then there are positive numbers $h_{0}=h_{0}(u, T)$ and $C=C(u, T)$ such that, for $h<h_{0}$, we have

$$
\left\|u_{h}(t)-u(t)\right\|+h\left\|u_{h}(t)-u(t)\right\|_{1} \leqslant C h^{r}, \text { for } \quad 0 \leqslant t \leqslant T .
$$

By the above continuation argument the theorem will follow once we have proved the following lemma.

Lemma 4. In addition to the assumptions of Theorem 2, assume that, for some $t_{1}$ with $0<t_{1}<T$, we have

$$
\|e\|_{L_{\infty}\left(0, t_{1} ; L_{2}\right)} \leqslant h^{r-1 / 4} \text {. }
$$


Then it follows that

$$
\|e\|_{L_{\infty}\left(0, t_{1} ; L_{2}\right)}+h\|e\|_{L_{\infty}\left(0, t_{1} ; H^{1}\right)} \leqslant C h^{r},
$$

where $C=C(u, T)$ does not depend on $t_{1}$.

Proof. Our assumption (3.15) implies that the bounds in (3.3), (3.5) and (3.6) hold. Considering first the case of $r>2$, we take $\chi=\theta$ in equation (3.12) to obtain

$$
\left(\theta_{\mathrm{l}}, \theta\right)+\left(a\left(u_{h}\right) \nabla \theta, \nabla \theta\right)=\left(R_{1}+R_{2}+R_{5}, \theta\right)+\left(R_{6}+R_{7}, \nabla \theta\right),
$$

now that $R_{3}=R_{4}=0$. Hence, by (2.1),

$$
\frac{\mathrm{d}}{\mathrm{d} t}\left(\|\theta\|^{2}\right)+\|\theta\|_{1}^{2} \leqslant C \sum_{\substack{j=1 \\ j \neq 3.4}}^{7}\left\|R_{j}\right\|^{2}+\|\theta\|_{1}^{2},
$$

so that, after integration with respect to $t$,

$$
\begin{aligned}
\|\theta(t)\|^{2} & \leqslant\|\theta(0)\|^{2}+C \sum_{\substack{j=1 \\
j \neq 3,4}}^{7}\left\|R_{j}\right\|_{L_{2}\left(0, t ; L_{2}\right)}^{2} \\
& \leqslant C h^{2 r}+C\|\theta\|_{L_{2}\left(0, t ; L_{2}\right)}^{2}, \text { for } 0<t<t_{1},
\end{aligned}
$$

where we have used the fact that

$$
\|\theta(0)\|=\left\|v_{h}-\tilde{v}_{h}\right\| \leqslant\left\|v_{h}-v\right\|+\left\|\tilde{v}_{h}-v\right\| \leqslant C h^{r},
$$

by (3.14) and (2.8), and simple modifications of the bounds of the terms $R_{i}$ derived in the proof of Lemma 3. Now an application of Grönwall's inequality shows

$$
\|\theta(t)\| \leqslant C h^{r}, \text { for } 0 \leqslant t \leqslant t_{1},
$$

and, by an inverse inequality, it hence follows that

$$
\|\theta(t)\|_{1} \leqslant C h^{r-1}, \text { for } 0 \leqslant t \leqslant t_{1} .
$$

Together with the appropriate bounds of $\bar{u}_{h}-u$ from Lemma 2 , this proves the desired result for $r>2$. The proof for the case $r=2$ is based on equation (3.13). This completes the proof.

\section{Analysis of the first method}

We shall now estimate the error in the approximation $u_{h}$ given by the semidiscrete problem: find $u_{n}:[0, T] \rightarrow S_{0 h}$ such that

$$
\begin{aligned}
\left(I_{h} H\left(u_{h}\right)_{t}, \chi\right)+\left(\nabla I_{h} G\left(u_{h}\right), \nabla \chi\right) & =\left(I_{h} f\left(u_{h}\right), \chi\right), \text { for } \chi \in S_{0 k}, 0<t<T, \\
u_{h}(0) & =v_{h} .
\end{aligned}
$$

We have the following result.

Theorem 3. Let $r \geqslant 3$ and let $u_{h}$ and $u$ be the solutions of (4.1) and (1.1), respectively, and assume that

$$
\left\|v_{h}-v\right\|+h\left\|v_{h}-v\right\|_{1} \leqslant C h^{r-1} .
$$


Then there are positive numbers $h_{0}=h_{0}(u, T)$ and $C=C(u, T)$ such that, for $h<h_{0}$, we have

$$
\begin{aligned}
& \left\|u_{h}(t)-u(t)\right\|+h\left\|u_{h}(t)-u(t)\right\|_{1}+\left(\int_{0}^{t}\left\|u_{h}(\tau)-u(\tau)\right\|_{1}^{2} \mathrm{~d} \tau\right)^{1 / 2} \leqslant C h^{r-1}, \\
& \text { for } 0 \leqslant t \leqslant T .
\end{aligned}
$$

Note that the above $L_{2}\left(0, T ; H^{1}\right)$ error bound is of optimal order, whereas the other bounds are less than optimal. The theorem will follow as before from the following lemma.

Lemma 5. In addition to the assumptions of Theorem 3, assume that, for some $t_{1}$ with $0<t_{1} \leqslant T$, we have

$$
\begin{aligned}
& \|e\|_{L_{\infty}\left(0, t_{1} ; L_{2}\right)} \leqslant h^{r-1-1 / 4}, \\
& \|e\|_{L_{2}\left(0, t_{1} ; H^{1}\right)} \leqslant h^{r-1-1 / 4} \\
& \left\|e_{l}\right\|_{L_{2}\left(0, t_{1} ; L_{2}\right)} \leqslant h^{r-2-1 / 4} .
\end{aligned}
$$

Then it follows that, for $h<h_{1}$,

$$
\text { and } \begin{aligned}
& \|e\|_{L_{\infty}\left(0, t_{1} ; L_{2}\right)}+\|e\|_{L_{2}\left(0, t_{1} ; H^{1}\right)} \leqslant C h^{r-1} \\
& \|e\|_{L_{\infty}\left(0, t_{1} ; H^{1}\right)}+\left\|e_{1}\right\|_{L_{2}\left(0, t_{1} ; L_{2}\right)} \leqslant C h^{r-2}
\end{aligned}
$$

where $h_{1}=h_{1}(u, T)$ and $C=C(u, T)$ do not depend on $t_{1}$.

Proof. In the same way as in our previous proofs the assumption (4.3) can be used to show that (remember that $r \geqslant 3$ and $d \leqslant 3$ )

$$
\begin{aligned}
& h\left\|u_{h}\right\|_{L_{\infty}\left(0, t_{1} ; w_{4}^{-1}\right)} \leqslant C, \\
& h^{1 / 4}\left\|u_{h}\right\|_{L_{\infty}\left(0, \ell_{1} ; W_{6}^{r-2}\right)} \leqslant C, \\
& \left\|u_{h}\right\|_{L_{\infty}\left(0, t_{1} ; W_{4}^{r-2}\right)} \leqslant C
\end{aligned}
$$

and

$$
\left\|u_{h}\right\|_{L_{\infty}\left(0, t_{1} ; W_{\infty}^{r-3}\right)} \leqslant C
$$

Similarly, (4.4) leads to

$$
\begin{aligned}
& \left\|u_{h}\right\|_{L_{2}\left(0, t_{1} ; w_{4}^{\prime-1}\right)} \leqslant C, \\
& \left\|u_{h}\right\|_{L_{2}\left(0, \ell_{1} ; W_{\infty}^{\prime 2}\right)} \leqslant C
\end{aligned}
$$

and (4.5) implies

$$
\begin{aligned}
& h^{2}\left\|u_{h, t}\right\|_{L_{2}\left(0, t_{1} ; W_{4}^{\prime-1}\right)} \leqslant C, \\
& h^{5 / 4}\left\|u_{h, l}\right\|_{L_{2}\left(0, t_{1} ; W_{6}^{r-2}\right)} \leqslant C, \\
& h\left\|u_{h, l}\right\|_{L_{2}\left(0, t_{1} ; W_{4}^{r-2}\right)} \leqslant C, \\
& h^{3 / 4}\left\|u_{h, l}\right\|_{L_{2}\left(0, r_{1} ; w_{\infty}^{r-3}\right)} \leqslant C .
\end{aligned}
$$


From these bounds it follows that

$$
\begin{aligned}
& \left\|f\left(u_{h}\right)\right\|_{L_{2}\left(0, t_{1} ; H^{\prime}\right)}+\left\|G\left(u_{h}\right)\right\|_{L_{2}\left(0, t_{1} ; H^{\prime}\right)}+\left\|H\left(u_{h}\right)\right\|_{L_{2}\left(0, t_{1} ; H^{\prime}\right)} \leqslant C, \\
& h\left\|H\left(u_{h}\right)\right\|_{L_{\infty}\left(0, t_{1} ; H^{\prime}\right)} \leqslant C, \\
& h^{2}\left\|H\left(u_{h}\right)_{t}\right\|_{L_{2}\left(0, t_{1} ; H^{\prime}\right)} \leqslant C .
\end{aligned}
$$

For example, for $r=3$-the most complicated case-we have, in view of (3.10) and since $\left\|u_{h}\right\|_{L_{\infty}\left(0, t_{1} ; L_{\infty}\right)} \leqslant C$ by $(4.8)$,

and

$$
\begin{aligned}
\left\|f\left(u_{h}\right)\right\|_{L_{2}\left(0, t_{1} ; H^{3}\right)} & \leqslant C\left(\left\|u_{h}\right\|_{L_{\infty}\left(0, t_{1} ; W_{4}^{1}\right)}\left\|u_{h}\right\|_{L_{2}\left(0, t_{1} ; W_{4}^{2}\right)}\right. \\
& \left.+\left\|u_{h}\right\|_{L_{\infty}\left(0, t_{1} ; W_{l}^{1}\right)}^{2}\left\|u_{h}\right\|_{L_{2}\left(0, t_{1} ; W_{\infty}^{1}\right)}\right) \leqslant C
\end{aligned}
$$

$$
h\left\|H\left(u_{h}\right)\right\|_{L_{\infty}\left(0, t_{1} ; H^{3}\right)} \leqslant C h\left(\left\|u_{h}\right\|_{L_{\infty}\left(0, t_{2} ; W_{4}^{1}\right)}\left\|u_{h}\right\|_{L_{\infty}\left(0, t_{1} ; W_{4}^{2}\right)}+\left\|u_{h}\right\|_{L_{\infty}\left(0, t_{1} ; W_{6}^{1}\right)}^{3}\right) \leqslant C .
$$

Similarly, in view of (3.11),

$$
\begin{aligned}
& h^{2}\left\|H\left(u_{h}\right)_{l}\right\|_{L_{2}\left(0, t_{1} ; H^{3}\right)}=h^{2}\left\|c\left(u_{h}\right) u_{h, t}\right\|_{L_{2}\left(0, t_{1} ; H^{3}\right)} \\
& \leqslant \\
& \quad+h^{2}\left(\left\|u_{h, t}\right\|_{L_{2}\left(0, t_{1} ; L_{\infty}\right)}\left(\left\|u_{h}\right\|_{L_{\infty}\left(0, t_{1} ; W_{4}^{1}\right)}\left\|u_{h}\right\|_{L_{\infty}\left(0, t_{1} ; W_{4}^{2}\right)}+\left\|u_{h}\right\|_{L_{\infty}\left(0, t_{1} ; W_{6}^{1}\right)}^{3}\right)\right. \\
& \quad+\left\|u_{h, l}\right\|_{L_{2}\left(0, t_{1} ; W_{4}^{2}\right)}\left\|u_{h}\right\|_{L_{\infty}\left(0, t_{1} ; W_{4}^{2}\right)}+\left\|u_{h, l}\right\|_{L_{2}\left(0, t_{1} ; W_{6}^{\prime}\right)}\left\|u_{h}\right\|_{L_{\infty}\left(0, t_{1} ; W_{6}^{1}\right)}^{2} \\
& \left.\quad+\left\|u_{h, l}\right\|_{L_{2}\left(0, t_{1} ; W_{4}^{2}\right)}\left\|u_{h}\right\|_{L_{\infty}\left(0, t_{1} ; W_{4}^{1}\right)}\right) \leqslant C .
\end{aligned}
$$

Similar arguments apply for $r \geqslant 4$.

We are now ready for the proof of the error estimates. With $\theta=u_{h}-\tilde{u}_{h}$ as before, we now have

$$
\begin{aligned}
& \left(c\left(u_{h}\right) \theta_{t}, \chi\right)+\left(a\left(u_{h}\right) \nabla \theta, \nabla \chi\right)=\left(\left(I_{h}-I\right) f\left(u_{h}\right), \chi\right)+\left(f\left(u_{h}\right)-f(u), \chi\right) \\
& -\left(\left(I_{h}-I\right) H\left(u_{h} h_{h}, \chi\right)-\left(\left(c\left(u_{h}\right)-c(u)\right) u_{t}, \chi\right)-\left(c\left(u_{h}\right)\left(\tilde{u}_{h, t}-u_{t}\right), \chi\right)\right. \\
& -\left(\nabla\left(I_{h}-I\right) G\left(u_{h}\right), \nabla \chi\right)-\left(\left(a\left(u_{h}\right)-a(u)\right) \nabla \tilde{u}_{h}, \nabla \chi\right) \\
& =\sum_{i=1}^{5}\left(R_{i}, \chi\right)+\sum_{i=6}^{7}\left(R_{i}, \nabla \chi\right),
\end{aligned}
$$

for $\chi \in S_{0 h}$ and $0<t<t_{1}$. Note that, except for the sixth term on the right-hand side, this equation is the same as (3.12). We begin with the proof of (4.7). To that end we take. $\chi=\theta_{t}$ in (4.12) to obtain

$$
\begin{aligned}
\left(c\left(u_{h}\right) \theta_{1}, \theta_{t}\right)+\frac{1}{2} \frac{\mathrm{d}}{\mathrm{d} t}\left(a\left(u_{h}\right) \nabla \theta, \nabla \theta\right)= & \sum_{i=1}^{5}\left(R_{i}, \theta_{t}\right)+\left(R_{6}, \nabla \theta_{t}\right)+\frac{\mathrm{d}}{\mathrm{d} t}\left(R_{7}, \nabla \theta\right) \\
& -\left(R_{7, v}, \nabla \theta\right)+\left(R_{8}, \nabla \theta\right),
\end{aligned}
$$

where $R_{8}=1 / 2 a\left(u_{h}\right)_{i} \nabla \theta$. Arguing similarly to the proof of Lemma 3, but applying an inverse inequality to the sixth term, we have

$$
\begin{aligned}
\int_{0}^{t}\left\|\theta_{t}\right\|^{2} \mathrm{~d} \tau+\|\theta(t)\|_{1}^{2} \leqslant & C\|\theta(0)\|_{1}^{2}+C \int_{0}^{t}\left(\sum_{i=1}^{5}\left\|R_{i}\right\|+h^{-1}\left\|R_{6}\right\|\right)\left\|\theta_{t}\right\| \mathrm{d} \tau \\
& +C\left\|R_{7}(t)\right\|\|\theta(t)\|_{1}+C\left\|R_{7}(0)\right\|\|\theta(0)\|_{1} \\
& +C \int_{0}^{t}\left(\left\|R_{7, t}\right\|+\left\|R_{8}\right\|\right)\|\theta\|_{1} \mathrm{~d} \tau,
\end{aligned}
$$


so that, after trivial estimates and a simple kick-back argument,

$$
\begin{aligned}
\left\|\theta_{t}\right\|_{L_{2}\left(0, t, L_{2}\right)}^{2}+\|\theta\|_{L_{\infty}\left(0, t, H^{1}\right)}^{2} \leqslant & C\|\theta(0)\|_{1}^{2}+C \sum_{i=1}^{5}\left\|R_{i}\right\|_{L_{2}\left(0, t, L_{2}\right)}^{2} \\
& +C h^{-2}\left\|R_{6}\right\|_{L_{2}\left(0, t, L_{2}\right)}^{2}+C\left\|R_{7}\right\|_{L_{\infty}\left(0, t ; L_{2}\right)}^{2} \\
& +C \int_{0}^{t}\left\|R_{7, t}\right\|\|\theta\|_{1} \mathrm{~d} \tau+C \int_{0}^{t}\left\|R_{8}\right\|\|\theta\|_{3} \mathrm{~d} \tau .
\end{aligned}
$$

For the first nine terms on the right-hand side we apply the same arguments as in the proof of Lemma 3. In view of (4.2), (4.9) and (4.11) these terms are thus bounded by

$$
C h^{2 r-4}+C\|\theta\|_{L_{2}\left(0, r, H^{1}\right)}^{2}+\varepsilon\|\theta\|_{L_{\infty}\left(0, t ; H^{1}\right)}^{2}+\varepsilon\left\|\theta_{t}\right\|_{L_{2}\left(0, t ; L_{2}\right)}^{2} .
$$

For the last term we use $u_{h, t}=\tilde{u}_{h, t}+\theta_{t}$ and Lemma 2 to obtain

$$
\begin{aligned}
\int_{0}^{t}\left\|R_{8}\right\|\|\theta\|_{1} \mathrm{~d} \tau \leqslant & C\left\|\tilde{u}_{h, t}\right\|_{L_{\infty}\left(0, t ; L_{\infty}\right)}\|\theta\|_{L_{2}\left(0, t ; H^{1}\right)}^{2} \\
& +C\left\|\theta_{t}\right\|_{L_{2}\left(0, t ; L_{2}\right)}\|\theta\|_{L_{2}\left(0, t ; W_{\infty}^{1}\right)}\|\theta\|_{L_{\infty}\left(0, t ; H^{1}\right)} \\
\leqslant & C\|\theta\|_{L_{2}\left(0, r ; H^{1}\right)}^{2}+C h^{1 / 4}\left\|\theta_{t}\right\|_{L_{2}\left(0, r, L_{2}\right)}\|\theta\|_{L_{\infty}\left(0, r ; H^{1}\right)} \\
\leqslant & C\|\theta\|_{L_{2}\left(0, t, H^{1}\right)}^{2}+\varepsilon\|\theta\|_{L_{\infty}\left(0, t ; H^{1}\right)}^{2}+\varepsilon\left\|\theta_{t}\right\|_{L_{2}\left(0, t ; L_{2}\right)}^{2}
\end{aligned}
$$

for $h$ small. Here we have also used the fact that

$$
\|\theta\|_{L_{2}\left(0, t ; W_{\infty}^{1}\right)} \leqslant C h^{-d / 2}\|\theta\|_{L_{2}\left(0, t ; H^{2}\right)} \leqslant C h^{-d / 2} \cdot C h^{r-1-1 / 4} \leqslant C h^{1 / 4} \text {, }
$$

by an inverse inequality and (4.4). Thus, altogether we now have

$$
\left\|\theta_{t}\right\|_{L_{2}\left(0, t ; L_{2}\right)}^{2}+\|\theta\|_{L_{\infty}\left(0, t ; H^{1}\right)}^{2} \leqslant C h^{2 r-4}+\|\theta\|_{L_{2}\left(0, t ; H^{1} b\right)}^{2} \text { for } 0<t<t_{1} \text {, }
$$

which by Grönwall's inequality and Lemma 2 proves (4.7).

We now turn to the proof of (4.6). Taking $\chi=\theta$ in (4.12) we have

$$
\begin{aligned}
& \frac{1}{2} \frac{\mathrm{d}}{\mathrm{d} t}\left(c\left(u_{h}\right) \theta, \theta\right)+\left(a\left(u_{h}\right) \nabla \theta, \nabla \theta\right)=\left(\left(I_{h}-I\right) f\left(u_{h}\right), \theta\right)+\left(f\left(u_{h}\right)-f(u), \theta\right) \\
& \quad-\frac{\mathrm{d}}{\mathrm{d} t}\left(\left(I_{h}-I\right) H\left(u_{h}\right), \theta\right)+\left(\left(I_{h}-I\right) H\left(u_{h}\right), \theta_{t}\right)-\left(\left(c\left(u_{h}\right)-c(u)\right) u_{t}, \theta\right) \\
& \quad-\left(c\left(u_{h}\right)\left(\tilde{u}_{h, t}-u_{t}\right), \theta\right)-\left(\nabla\left(I_{h}-I\right) G\left(u_{h}\right), \nabla \theta\right)-\left(\left(a\left(u_{h}\right)-a(u)\right) \nabla \tilde{u}_{h}, \nabla \theta\right) \\
& \quad+\frac{1}{2}\left(c\left(u_{h}\right)_{t} \theta, \theta\right) \\
& =\left(R_{1}+R_{2}+R_{4}+R_{5}, \theta\right)+\left(R_{6}+R_{7}, \nabla \theta\right)+\frac{\mathrm{d}}{\mathrm{d} t}\left(\tilde{R}_{3}, \theta\right)-\left(\tilde{R}_{3}, \theta_{t}\right)+\left(R_{9}, \theta\right),
\end{aligned}
$$

where $\tilde{R}_{3}=-\left(I_{h}-I\right) H\left(u_{h}\right)$ and $R_{9}=1 / 2 c\left(u_{h}\right)_{h} \theta$ and the other terms are the same as above. By simple estimates and integration with respect to $t$ we obtain

$$
\begin{aligned}
& \|\theta\|_{L_{\infty}\left(0, t ; L_{2}\right)}^{2}+\|\theta\|_{L_{2}\left(0, t ; H^{1}\right)}^{2} \leqslant C\|\theta(0)\|^{2}+C\left(\sum_{i=1}^{2}+\sum_{i=4}^{7}\right)\left\|R_{i}\right\|_{L_{2}\left(0, t ; L_{2}\right)}^{2} \\
& +C\left\|\tilde{R}_{3}\right\|_{L_{\infty}\left(0, t ; L_{2}\right)}^{2}+C\left\|\tilde{R}_{3}\right\|_{L_{2}\left(0, t ; L_{2}\right)}\left\|\theta_{t}\right\|_{L_{2}\left(0, t ; L_{2}\right)}+C \int_{0}^{t}\left\|R_{9}\right\|\|\theta\| \mathrm{d} \tau .
\end{aligned}
$$


By (4.2) and in view of (4.8) and (4.9), the first five terms on the right-hand side can be bounded by

$$
C h^{2 r-2}+C\|\theta\|_{L_{2}\left(0, r ; L_{2}\right)}^{2}
$$

just as in the proof of Lemma 3. Also, from (4.9) it follows that

$$
\left\|R_{6}\right\|_{L_{2}\left(0, t ; L_{2}\right)} \leqslant C h^{r-1}\left\|G\left(u_{h}\right)\right\|_{L_{2}\left(0, t ; H^{r}\right)} \leqslant C h^{r-1} \text {. }
$$

For the seventh term we have

$$
\begin{aligned}
\left\|R_{7}\right\|_{L_{2}\left(0, t ; L_{2}\right)} & \leqslant C\left\|u_{h}-u\right\|_{L_{2}\left(0, t ; L_{2}\right)}\left\|\tilde{u}_{h}\right\|_{L_{\infty}\left(0, t ; W_{\infty}^{1}\right)} \\
& \leqslant C\left(\|\theta\|_{L_{2}\left(0, t ; L_{2}\right)}+\left\|\tilde{u}_{h}-u\right\|_{L_{2}\left(0, t ; L_{2}\right)}\right) \\
& \leqslant C h^{r}+C\|\theta\|_{L_{2}\left(0, t ; L_{2}\right)} .
\end{aligned}
$$

Further, using (4.10) we have

$$
\left\|\tilde{R}_{3}\right\|_{L_{\infty}\left(0, t ; L_{2}\right)} \leqslant C h^{r}\left\|H\left(u_{h}\right)\right\|_{L_{\infty}\left(0, t ; H^{\prime}\right)} \leqslant C h^{r-1}
$$

and, by (4.9) and the already proven error bound (4.7),

$$
\begin{aligned}
\left\|\tilde{R}_{3}\right\|_{L_{2}\left(0, t ; L_{2}\right)}\left\|\theta_{t}\right\|_{L_{2}\left(0, t ; L_{2}\right)} & \leqslant C h^{r}\left\|H\left(u_{k}\right)\right\|_{L_{2}\left(0, t ; H^{r}\right)}\left\|\theta_{t}\right\|_{L_{2}\left(0, t ; L_{2}\right)} \\
& \leqslant C h^{r} \cdot C h^{r-2}=C h^{2 r-2} .
\end{aligned}
$$

For the last term we write $u_{h_{,} t}=u_{t}+e_{\imath}$ to obtain

$$
\begin{aligned}
\int_{0}^{t}\left\|R_{9}\right\|\|\theta\| \mathrm{d} \tau \leqslant & C\left\|u_{t}\right\|_{L_{\infty}\left(0, t ; L_{\infty}\right)}\|\theta\|_{L_{2}\left(0, t ; L_{2}\right)}^{2} \\
& +C\left\|e_{t}\right\|_{L_{2}\left(0, t ; L_{2}\right)}\|\theta\|_{L_{2}\left(0, t ; L_{\infty}\right)}\|\theta\|_{L_{\infty}\left(0, t ; L_{2}\right)} \\
\leqslant & C\|\theta\|_{L_{2}\left(0, t ; L_{2}\right)}^{2}+C h^{1 / 4}\|\theta\|_{L_{2}\left(0, t ; H^{1}\right)}\|\theta\|_{L_{\infty}\left(0, t ; L_{2}\right)} \\
\leqslant & \leqslant\|\theta\|_{L_{2}\left(0, t ; L_{2}\right)}^{2}+\varepsilon\|\theta\|_{L_{2}\left(0, t ; H^{1}\right)}^{2}+\varepsilon\|\theta\|_{L_{\infty}\left(0, t ; L_{2}\right)}^{2}
\end{aligned}
$$

for $h$ small. Here we also used the fact that

$$
\begin{aligned}
\left\|e_{t}\right\|_{L_{2}\left(0, t ; L_{2}\right)}\|\theta\|_{L_{2}\left(0, t ; L_{\infty}\right)} & \leqslant C h^{r-2}\|\theta\|_{L_{2}\left(0, t ; W_{4}^{1}\right) .} \\
& \leqslant C h^{r-2} \cdot C h^{-d / 4}\|\theta\|_{L_{2}\left(0, t ; H^{1}\right)} \\
& \leqslant C h^{1 / 4}\|\theta\|_{L_{2}\left(0, t ; H^{1}\right)},
\end{aligned}
$$

by (4.7), Sobolev's inequality and an inverse inequality. Summing up, we now have

$$
\|\theta\|_{L_{\infty}\left(0, t ; L_{2}\right)}^{2}+\|\theta\|_{L_{2}\left(0, t ; H^{1}\right)}^{2} \leqslant C h^{2 r-2}+C\|\theta\|_{L_{2}\left(0, t ; L_{2}\right)}^{2} \text {, for } 0<t<t_{1},
$$

and (4.6) follows, which completes the proof of the lemma.

In the previous theorem we have presented a suboptimal order estimate for the $L_{2}$ norm of the error. Naturally, one might ask about the possibility of obtaining an estimate of optimal order of accuracy. We shall give a positive answer in two cases. The first is the case where $c(u) \equiv 1$ and $r \geqslant 3, d \leqslant 3$ and the error is measured in the $L_{2}\left(0, T ; L_{2}\right)$ norm. The other is the case of $r=2, d=1$, where we obtain a bound in the $L_{\infty}\left(0, T, L_{2}\right)$ norm for general $c=c(u)$. 
Theorem 4. Let $c(u) \equiv 1$ and $r \geqslant 3, d \leqslant 3$ and let $u_{h}$ and $u$ be the solutions of (4.1) and (1.1), respectively, and assume that

$$
\left\|v_{h}-v\right\| \leqslant C h^{r}
$$

Then there are positive numbers $h_{0}=h_{0}(u, T)$ and $C=C(u, T)$ such that, for $h<h_{0}$, we have

$$
h\left\|u_{h}(t)-u(t)\right\|+\left(\int_{0}^{t}\left\|u_{h}(\tau)-u(\tau)\right\|^{2} \mathrm{~d} \tau\right)^{1 / 2} \leqslant C h^{r}, \text { for } 0 \leqslant t \leqslant T .
$$

The theorem follows as before from the following lemma.

Lemma 6. In addition to the assumptions of Theorem 4, assume that, for some $t_{1}$ with $0<t_{1} \leqslant T$, we have

$$
\begin{aligned}
& \|e\|_{L_{\infty}\left(0, t_{1} ; L_{2}\right)} \leqslant h^{r-1-1 / 4}, \\
& \|e\|_{L_{2}\left(0, t_{1} ; L_{2}\right)} \leqslant h^{r-1 / 4} .
\end{aligned}
$$

Then it follows that, for $h<h_{1}$,

$$
\begin{aligned}
& \|e\|_{L_{\infty}\left(0, t_{1} ; L_{2}\right)} \leqslant C h^{r-1}, \\
& \|e\|_{L_{2}\left(0, t_{1} ; L_{2}\right)} \leqslant C h^{r},
\end{aligned}
$$

where $h_{1}=h_{1}(u, T)$ and $C=C(u, T)$ do not depend on $t_{1}$.

Proof. Just as in the proof of Lemma 5 for $r \geqslant 3, d \leqslant 3$ we find that the bounds (4.8) and (4.9) follow from the assumptions (4.14) and (4.15). Moreover, the error bound (4.16) was already proved in Lemma 5.

Since $c(u) \equiv 1$, the error equation (4.12) now becomes

$$
\begin{aligned}
& \left(\theta_{t}, \chi\right)+(a(u) \nabla \theta, \nabla \chi)=\left(\left(I_{h}-I\right) f\left(u_{h}\right), \chi\right)+\left(f\left(u_{h}\right)-f(u), \chi\right) \\
& \quad-\left(\tilde{u}_{h, z}-u_{t}, \chi\right)-\left(\nabla\left(I_{h}-I\right) G\left(u_{h}\right), \nabla \chi\right)-\left(\left(a\left(u_{h}\right)-a(u)\right) \nabla u_{h}, \nabla \chi\right) \\
& =\sum_{i=1}^{3}\left(S_{i}, \chi\right)+\sum_{i=4}^{5}\left(S_{i}, \nabla \chi\right),
\end{aligned}
$$

for $\chi \in S_{0 h}$ and $0<t<t_{1}$. Note that we have, for later convenience, replaced $a\left(u_{h}\right)$ in the second term on the left by $a(u)$, which implies a modification of the fifth term on the right.

To prepare for the proof of (4.17) we recall the operators $T$ and $T_{h}$ defined in Section 2. It is well known that $T_{h}$ is a self-adjoint, positive semidefinite bounded operator on $L_{2}(\Omega)$, which is positive definite on $S_{0 h}$. We shall use the equivalence of norms

$$
\left(\chi, T_{h} \chi\right)^{1 / 2} \cong\left\|T_{h} \chi\right\|_{1}, \quad \chi \in S_{0 h},
$$

which immediately follows from (2.5) in view of the positivity and boundedness of $a(u)$.

After these preparations we now set $\chi=T_{h} \theta$ in (4.19). We have

$$
\frac{1}{2} \frac{\mathrm{d}}{\mathrm{d} t}\left(\theta, T_{h} \theta\right)+\|\theta\|^{2}=\sum_{i=1}^{3}\left(S_{i}, T_{h} \theta\right)+\sum_{i=4}^{5}\left(S_{i}, \nabla T_{h} \theta\right)+\left(S_{6}, \theta\right)
$$

where $S_{6}=1 / 2 T_{h, t} \theta$. By integration and simple estimates, using (4.20) and the boundedness of $T_{h}$, we obtain 


$$
\begin{array}{rl}
\left\|T_{h} \theta\right\|_{L_{\infty}\left(0, t ; H^{1}\right)}^{2}+\|\theta\|_{L_{2}\left(0, r ; L_{2}\right) \leqslant}^{2} & C\|\theta(0)\|^{2}+C \sum_{i=1}^{3} \int_{0}^{t}\left(S_{i}, T_{h} \theta\right) \mathrm{d} \tau \\
& +C \sum_{i=4}^{5} \int_{0}^{t}\left(S_{i}, \nabla T_{h} \theta\right) \mathrm{d} \tau+C \int_{0}^{t}\left(S_{6}, \theta\right) \mathrm{d} \tau .
\end{array}
$$

In view of (4.13) the first term on the right is bounded by $\mathrm{Ch}^{2 r}$. Next we have

because

$$
\begin{aligned}
\sum_{i=1}^{3} \int_{0}^{t}\left(S_{i}, T_{h} \theta\right) \mathrm{d} \tau & \leqslant \sum_{i=1}^{3} \int_{0}^{t}\left(S_{i}, T_{h} S_{i}\right)^{1 / 2}\left(\theta, T_{h} \theta\right)^{1 / 2} \mathrm{~d} \tau \\
& \leqslant C \sum_{i=1}^{3} \int_{0}^{t}\left\|S_{i}\right\|\left\|T_{h} \theta\right\|_{1} \mathrm{~d} \tau \\
& \leqslant C \sum_{i=1}^{3}\left\|S_{i}\right\|_{L_{2}\left(0, t ; L_{2}\right)}\left\|T_{h} \theta\right\|_{L_{2}\left(0, t ; H^{1}\right)} \\
& \leqslant C h^{2 r}+\varepsilon\|\theta\|_{L_{2}\left(0, t ; L_{2}\right)}^{2}+C\left\|T_{h} \theta\right\|_{L_{2}\left(0, t ; H^{1}\right)}^{2}
\end{aligned}
$$

$$
\begin{aligned}
& \left\|S_{1}\right\|_{L_{2}\left(0, r ; L_{2}\right)} \leqslant C h^{r}\left\|f\left(u_{h}\right)\right\|_{L_{2}\left(0, t ; H^{r}\right)} \leqslant C h^{r}, \\
& \left\|S_{2}\right\|_{L_{2}\left(0, t ; L_{2}\right)} \leqslant C\left\|u_{h}-u\right\|_{L_{2}\left(0, t ; L_{2}\right)} \leqslant C h^{r}+C\|\theta\|_{L_{2}\left(0, t ; L_{2}\right)}, \\
& \left\|S_{3}\right\|_{L_{2}\left(0, t ; L_{2}\right)} \leqslant C h^{r},
\end{aligned}
$$

by (2.2), (4.9), (2.8) and (4.18). Further

$$
\begin{aligned}
\int_{0}^{t}\left(S_{4}, \nabla T_{h} \theta\right) \mathrm{d} \tau= & -\int_{0}^{t}\left(\nabla\left(I_{h}-I\right) G\left(u_{h}\right), \nabla T_{h} \theta\right) \mathrm{d} \tau \\
= & -\int_{0}^{t}\left(\nabla\left(I_{h}-I\right) G\left(u_{h}\right), \nabla\left(T_{h}-T\right) \theta\right) \mathrm{d} \tau \\
& +\int_{0}^{t}\left(\left(I_{h}-I\right) G\left(u_{h}\right), \Delta T \theta\right) \mathrm{d} \tau \\
\leqslant & \int_{0}^{t}\left\|\left(I_{h}-I\right) G\left(u_{h}\right)\right\|_{1}\left\|\left(T_{h}-T\right) \theta\right\|_{1} \mathrm{~d} \tau \\
& +\int_{0}^{t}\left\|\left(I_{h}-I\right) G\left(u_{h}\right)\right\|\|T \theta\|_{2} \mathrm{~d} \tau \\
\leqslant & C h^{r} \int_{0}^{t}\left\|G\left(u_{h}\right)\right\|_{r}\|\theta\| \mathrm{d} \tau \leqslant C h^{r}\left\|G\left(u_{h}\right)\right\|_{L_{2}\left(0, t ; H^{r}\right)}\|\theta\|_{L_{2}\left(0, t ; L_{2}\right)} \\
\leqslant & C h^{2 r}+\varepsilon\|\theta\|_{L_{2}\left(0, t ; L_{2}\right)},
\end{aligned}
$$

where we have used (2.2), (2.7), (2.6) and (4.9).

Next we use $u_{h}=\tilde{u}_{h}+\theta$ to write

$$
\begin{aligned}
& \int_{0}^{t}\left(S_{5}, \nabla T_{h} \theta\right) \mathrm{d} \tau=\int_{0}^{t}\left(\left(a\left(u_{h}\right)-a(u)\right) \nabla \tilde{u}_{h}, \nabla T_{h} \theta\right) \mathrm{d} \tau \\
& \quad+\int_{0}^{t}\left(\left(a\left(u_{h}\right)-a(u)\right) \nabla \theta, \nabla T_{h} \theta\right) \mathrm{d} \tau
\end{aligned}
$$


where, by (2.9) and (2.8),

$$
\begin{aligned}
& \int_{0}^{t}\left(\left(a\left(u_{h}\right)-a(u)\right) \nabla \tilde{u}_{h}, \nabla T_{h} \theta\right) \mathrm{d} \tau \leqslant C\|e\|_{L_{2}\left(0, t ; L_{2}\right)}\left\|\tilde{u}_{h}\right\|_{L_{\infty}\left(0, t ; W_{\infty}^{1}\right)}\left\|T_{h} \theta\right\|_{L_{2}\left(0, t ; H^{1}\right)} \\
& \leqslant C\left(\|\theta\|_{L_{2}\left(0, t ; L_{2}\right)}+\left\|\tilde{u}_{h}-u\right\|_{L_{2}\left(0, t ; L_{2}\right)}\right)\left\|T_{h} \theta\right\|_{L_{2}\left(0, t ; H^{1}\right)} \\
& \leqslant C h^{2 r}+\varepsilon\|\theta\|_{L_{2}\left(0, t ; L_{2}\right)}^{2}+C\left\|T_{h} \theta\right\|_{L_{2}\left(0, t ; H^{1}\right)}^{2},
\end{aligned}
$$

and, by virtue of Lemma 1 and (4.15) and in view of the restrictions on $r$ and $d$,

$$
\begin{aligned}
\int_{0}^{t}\left(\left(a\left(u_{h}\right)-a(u)\right) \nabla \theta, \nabla T_{h} \theta\right) \mathrm{d} \tau & \leqslant C\|e\|_{L_{2}\left(0, t ; L_{\infty}\right)}\|\theta\|_{L_{2}\left(0, t ; H^{1}\right)}\left\|T_{h} \theta\right\|_{L_{\infty}\left(0, t ; H^{1}\right)} \\
& \leqslant C h^{-(d / 2)+r-1 / 4} \cdot C h^{-1}\|\theta\|_{L_{2}\left(0, t ; L_{2}\right)}\left\|T_{h} \theta\right\|_{L_{\infty}\left(0, t ; H^{1}\right)} \\
& \leqslant C h^{1 / 4}\|\theta\|_{L_{2}\left(0, t ; L_{2}\right)}\left\|T_{h} \theta\right\|_{L_{\infty}\left(0, t ; H^{1}\right)} \\
& \leqslant \varepsilon\|\theta\|_{L_{2}\left(0, t ; L_{2}\right)}^{2}+\varepsilon\left\|T_{h} \theta\right\|_{L_{\infty}\left(0, t ; H^{1}\right)}^{2},
\end{aligned}
$$

for $h$ small. For the last term we have

$$
\begin{aligned}
\int_{0}^{t}\left(S_{6}, \theta\right) \mathrm{d} \tau=\frac{1}{2} \int_{0}^{t}\left(\theta, T_{h, t} \theta\right) \mathrm{d} \tau & =\frac{1}{2} \int_{0}^{t}\left(a(u) \nabla T_{h} \theta, \nabla T_{h, t} \theta\right) \mathrm{d} \tau \\
& =-\frac{1}{2} \int_{0}^{t}\left(a(u)_{t} \nabla T_{h} \theta, \nabla T_{h} \theta\right) \mathrm{d} \tau \\
& \leqslant C\left\|T_{h} \theta\right\|_{L_{2}\left(0, t ; H^{1}\right)}^{2}
\end{aligned}
$$

by (2.5) and by differentiation of (2.5) with respect to time.

Together these estimates show

$$
\left\|T_{h} \theta\right\|_{L_{\infty}\left(0, t ; H^{1}\right)}^{2}+\|\theta\|_{L_{2}\left(0, t ; L_{2}\right)}^{2} \leqslant C h^{2 r}+C\left\|T_{h} \theta\right\|_{L_{2}\left(0, t ; H^{1}\right)}^{2}, \quad 0 \leqslant t \leqslant t_{1},
$$

and Grönwall's lemma yields

$$
\left\|T_{h} \theta\right\|_{L_{\infty}\left(0, t_{1} ; H^{1}\right)}^{2}+\|\theta\|_{L_{2}\left(0, r_{1} ; L_{2}\right)}^{2} \leqslant C h^{2 r}
$$

which proves (4.17). The lemma is proved.

The case $r=2, d=1$ is somewhat special, because of the well-known fact that the interpolation operator $I_{h}$ then coincides with the standard Ritz projection $R_{h}^{0}$ : $H_{0}^{2}(\Omega) \rightarrow S_{0 h}$ defined by

$$
\left(\nabla\left(R_{h}^{0} w-w\right), \nabla \chi\right)=0, \quad \chi \in S_{0 h},
$$

(see, for instance, p. 80 of Reference 13). In the error equation (4.12) we therefore have $\left(R_{6}, \nabla \chi\right)=0$, so that (4.12) is identical to equation (3.12) in the proof of Lemma 3 with the corresponding term removed. Repeating the appropriate steps of the proof of Theorem 1, with the term $\left(R_{6}, \nabla \chi\right)$ removed, we therefore find that the second order convergence of the second method carries over to the present case. We thus have:

Theorem 5. Let $r=2, d=1$ and $u_{k}$ and $u$ be the solutions of (4.1) and (1.1), respectively, and assume that $v_{h}=\tilde{v}_{h}=\tilde{u}_{h}(0)$. Then there are positive numbers $h_{0}=h_{0}(u, T)$ and $C=C(u, T)$ such that, for $h<h_{0}$, we have

$$
\left\|u_{h}(t)-u(t)\right\|+h\left\|u_{h}(t)-u(t)\right\|_{1} \leqslant C h^{2}, \text { for } 0 \leqslant t \leqslant T .
$$




\section{Acknowledgement}

Part of this work was done during a visit by the first author (S. L.) to the Mathematical Sciences Institute at Cornell University. The hospitality of the institute is gratefully acknowledged.

\section{References}

1. Borshukova, S. and Konovski, $P$., 'Transformation of dependent variable in the finite element solution of some phase change problems', Int. j. numer. methods. eng., 20, 1815-1821 (1984).

2. Cermák, L. and Zlámal, M., 'Transformation of dependent variables and the finite element solution of nonlinear evolution equations', Int. j. numer. methods eng., 15, 31-40 (1980).

3. Christie, J., Griffiths, D. F., Mitchell, A. R. and Sanz-Serna, J. M., 'Product approximation for nonlinear problems in the finite element method', IMA J. Numer. Anal, 1, 253-266 (1981).

4. Ciarlet, P. G., The Finite Element Method for Elliptic Problems, North-Holland, 1978.

5. Douglas, J. Jr. and Dupont, T., 'The effect of interpolating the coefficients in nonlinear parabolic Galerkin procedures', Math. Comp., 29, 360-389 (1975).

6. Elliott, C. M., 'Error analysis of the enthalpy method for the Stefan problem', IMA J. Numer. Anal., 7, 61-71 (1987)

7. Grisvard, P., Elliptic Problems in Nonsmooth Domains, Pitman, 1985.

8. Khalsa, S. N. S., 'Finite element approximation of a reaction diffusion equation. Part I: Application of a topological technique to the analysis of asymptotic behavior of the semidiscrete equation', Quarterly Appl. Math., 44, 375-386 (1986).

9. Nie, Y.-Y. and Thomée, V., 'A lumped mass finite element method with quadrature for a nonlinear parabolic problem', IMA J. Numer. Anal., 5, 371-396 (1985).

10. Raviart, P. A., 'The use of numerical integration in finite element methods for solving parabolic equations', in Miller, J. J. H. (ed.), Topics in Numerical Analysis, Academic Press, 1973, pp. 233-264.

11. Sanz-Serna, J. M. and Abia, L., 'Interpolation of the coefficients in nonlinear elliptic Galerkin procedures', SIAM J. Numer. Anal., 21, 77-83 (1984).

12. Schatz, A. H. and Wahlbin, L. B., 'On the quasi-optimality in $L_{\infty}$ of the $\dot{H}^{1}$-projection into finite element spaces', Math. Comp., 38, 1-22 (1982).

13. Thomee, V., Galerkin Finite Element Methods for Parabolic Problems, Lecture Notes in Mathematics, Vol. 1054, Springer-Verlag, 1984. 